\title{
Outcome Comparison Between Tocilizumab Alone Vs Tocilizumab Followed by Intravenous Immunoglobulin (IVIG) Group in Critical COVID-19 Disease
}

\author{
Syeda Rida-e-Zehra ${ }^{1,}$, , Shamim Kausar ${ }^{1,2}$, Anum Latif ${ }^{1}$, Samar Abbas Jaffri ${ }^{1,2}$, \\ Syeda Namayah Fatima Hussain ${ }^{1}$, Irfan Ahsan ${ }^{1}$, Muhammad Rafay ${ }^{1}$, Pirih Bhatti ${ }^{1}$ \\ ${ }^{1}$ Department of Internal Medicine, Liaquat National Hospital and Medical College, Karachi, Pakistan \\ ${ }^{2}$ Department of Internal Medicine, National Medical Center, Karachi, Pakistan
}

Email address:

s.rida.e.zehra@gmail.com (S. Rida-e-Zehra)

${ }^{*}$ Corresponding author

\section{To cite this article:}

Syeda Rida-e-Zehra, Shamim Kausar, Anum Latif, Samar Abbas Jaffri, Syeda Namayah Fatima Hussain, Irfan Ahsan, Muhammad Rafay, Pirih Bhatti. Outcome Comparison Between Tocilizumab Alone vs Tocilizumab Followed by Intravenous Immunoglobulin (IVIG) Group in Critical COVID-19 Disease. International Journal of Infectious Diseases and Therapy. Vol. 6, No. 1, 2021, pp. 37-40. doi: $10.11648 /$ j.ijidt.20210601.15

Received: October 23, 2020; Accepted: January 18, 2021; Published: February 27, 2021

\begin{abstract}
Currently, COVID-19 is one of the most pressing healthcare problems across the world. With no definitive pharmacological guidelines, multiple drugs were used to treat critical patients with little success. of Tocilizumab, a monoclonal antibody has shown some role in the treatment of Covid-19 infection. The study was a cross-sectional prospective observational study. It was conducted in the COVID-19 Intensive Care Unit (ICU) of Liaquat National Hospital and Medical College, and National Medical Centre Karachi. The study was conducted from $15^{\text {th }}$ June 2020 to $31^{\text {st }}$ July 2020. Objective of the study was to compare the outcomes of severe to critical COVID-19 patients with established Cytokine release Syndrome (CRS), who received Tocilizumab with the group received Tocilizumab followed by intravenous immunoglobulins (IVIG). Two groups were made with one receiving Tocilizumab alone while the other received IVIG after Tocilizumab. Comparison was then made based on frequency of mortality as well the need of mechanical ventilation and its range of days. In results $4(15.4 \%)$ patients in Tocilizumab only group died while Tocilizumab followed by IVIG treated group had $10(38.46 \%)$ deaths. 20 (76.9\%) patients needed mechanical ventilation in Tocilizumab only group while Tocilizumab followed by IVIG treated group consisted of 23 $(88.46 \%)$ patients. This concludes that the group with only Tocilizumab therapy has better outcome as compare to the group who received both Tocilizumab and intravenous immunoglobulins.
\end{abstract}

Keywords: COVID-19, Coronavirus, Tocilizumab, Immunoglobulin

\section{Introduction}

A novel virus COVID -19 spread as pandemic in 2020 [1] Almost all countries of the world reported positive cases with different prevalence [2]. In some part of the world like Italy and China high mortality was observed [3-6]. Since treatment guidelines were not available in beginning many drug options were claimed to be beneficial for its management [7] Initially role of chloroquine and Azithromycin was proven as useful but later on its role became controversial and some studies proved it to be harmful [8-11]. Considering the pathophysiology of this disease and its pronounced effect on cytokine release many drugs with its potential effect on immune suppression were tried and amongst them Remdisivir showed significant impact on outcomes of patients [12-15]. However, it could not change the outcome of severe to critical grade patients. Role of steroids were considered in many critical patient and study showed it to be beneficial [16]. Many patients despite high dose of steroid therapy did not show much improvement in inflammatory markers for that role of Tocilizumab a monoclonal antibody emerged [16]. Many patients showed drastic improvement in declining inflammatory markers and clinical improvement of patients [17]. However, despite Tocilizumab many patients 
did not show significant improvement in CRS markers [18]. Role of Intra Venous Immunoglobulin (IVIG) is well defined in suppressing immune response in many autoimmune disorders $[19,20]$. In many cases of COVID-19 infections IVIG was given and it proved to be beneficial in suppressing cytokine release syndrome however due to its high cost it was not used as first line treatment in COVID-19 infection. There is very limited data of comparison of outcome in patients who received Tocilizumab vs Tocilizumab and IVIG. We aimed to compare these two groups outcome in critical care on patients with severe to critical COVID disease.

\section{Material and Methods}

The study was a cross-sectional prospective observational study. It was conducted in the COVID-19 Intensive Care Unit (ICU) of Liaquat National Hospital and Medical College, and National Medical Centre Karachi. The study was conducted from 15 th June 2020 to 31 st July 2020. Objective of the study was to compare the outcomes of severe to critical COVID-19 patients with established Cytokine release Syndrome (CRS), who received Tocilizumab with the group received Tocilizumab followed by IVIG. All patients with severe to critical confirmed COVID-19 infection through a detected nasopharyngeal swab Polymerase Chain Reaction (PCR), with PO2 to Fio2 ratio of $<200$, who had a serum ferritin level of $>1000$ and had already received high dose steroids ( $1 \mathrm{mg} / \mathrm{kg} /$ day of either Methylprednisolone or Prednisolone) were included in the study. Those patients who received plasma exchange therapy or convalescent plasma therapy excluded from study. Outcomes were defined as numbers of days on Mechanical Ventilation, days in critical care, mortality and survival for each comparison group. Tocilizumab group was considered for the participant who fulfilled inclusion criteria and received at least single dose of weight based standard regimen of Tocilizumab. IVIG group was considered for patients who received at least one dose of weight-based Tocilizumab followed by weight based standard regimen $400 \mathrm{mg} / \mathrm{kg} /$ once daily dose of IVIG for 5 days on persistently raised serum ferritin despite Tocilizumab treatment. Critical COVID-19 patients were defined as patients who diagnosed for Acute Respiratory Distress Syndrome (ARDS) with PF ratio of $<200$. Participants of study were divided in two groups based on treatment with Tocilizumab or Tocilizumab followed by IVIG treatment. Patients history regarding age and gender was taken. Confounding variables and biasness were controlled by strictly following the inclusion and exclusion criteria. Data was recorded in pre-formed proforma.

\section{Results}

Total 52 patients were included in this study. These patients were admitted in critical care unit with moderate to severe Covid pneumonia. These participants were divided into two groups, 26 patients in each group. Participants of group one received Tocilizumab only therapy and participants of group two received Tocilizumab therapy followed by IVIG. Outcome of these two groups were measured in terms of need of mechanical ventilation and mortality rate. Majority of the patients were male and average age was 56 years. Mean age of patients in Tocilizumab alone treated group was $62.31 \pm 11.471$ while in other group the mean age was 59.25 \pm 9.644 .

In Tocilizumab alone treated group $22(84.6 \%)$ patients had severe ARDS with a $\mathrm{P} / \mathrm{F}$ ratio $<100$. While $4(15.4 \%)$ of the patients had moderate ARDS with $\mathrm{P} / \mathrm{F}$ ratio ranging 200 to 100. In Tocilizumab followed by IVIG treated group 23 (88.46\%) patients had severe ARDS and 3 (11.53\%) patients were in moderate ARDS. Both groups have the patients with almost same severity of disease. Both groups were given their respective therapy and outcomes were noted with in the duration of one month.

Out of 26 patients in Tocilizumab alone treated group 20 $(76.9 \%)$ patients received mechanical ventilation and 6 (23.1\%) patients were managed on non-invasive ventilation. In other group $23(88.46 \%)$ patients received mechanical ventilation and $3(11.53 \%)$ patients were managed without mechanical ventilation.

In Tocilizumab alone treated group $4(15.4 \%)$ patients had mortalities while $22(84.6 \%)$ patients survived. In Tocilizumab followed by IVIG treated group 10 (38.46\%) patients had mortalities while $16(61.53 \%)$ patients survived.

Range of days on mechanical ventilation was $0-5$ in Tocilizumab alone treated group and it was $0-11$ in group treated with Tocilizumab followed by IVIG. These variables are summarized in table 1 below.

Table 1. Demography of the study.

\begin{tabular}{lll}
\hline Variable & Tocilizumab Only Group & Tocilizumab followed by IVIG Group \\
\hline No of participants & 26 & 26 \\
Patients with severe disease & 22 & 23 \\
Patients with moderate disease & 4 & 3 \\
Mean age in years & 62 & 59 \\
Patient received mechanical ventilation & $20(76.9 \%)$ & $23(88.46 \%)$ \\
Days of mechanical ventilation & $0-5$ & $0-11$ \\
Mortality Rate & $4(15.4 \%)$ & $10(38.46 \%)$ \\
\hline
\end{tabular}

\section{Discussion}

In COVID-19 infection, a surge in inflammatory markers was observed in those patients who had severe to critical COVID-19 infection [21]. Many treatments were tried when on steroids, patient continued to show worsening in inflammatory markers [22]. Pakistan being low income 
countries many treatment options like IVIG and Plasma therapy was not affordable by many critical patients despite worsening in inflammatory markers [23]. However, some of them managed to afford this treatment. IVIG with its well-established role in autoimmune diseases and suppression of bodies' immune response can help accelerate the recovery of patients [24]. Being a protein rich plasma product high in antibodies make it a valuable adjuvant treatment option. It has also been used in previous pandemics like Severe acute respiratory syndrome (SARS), Middle East Respiratory Syndrome (MERS) and 2009 H1N1 Pandemic with great results in the influenza disease [25-27]. In our study we observed that those patients who received only Tocilizumab were mostly affected by severe ARDS and only few of them had moderate ARDS. As compared to Tocilizumab followed by IVIG group more patients were affected by severe ARDS in Tocilizumab only group. In Tocilizumab alone treated group most of the patients were intubated and mechanically ventilated and same was observed in Tocilizumab followed by IVIG group. However, on comparison of both group our study showed that there was more preponderance of receiving mechanical ventilation in Tocilizumab followed by IVIG group. Comparing the mortalities between two groups our study proved that those patients who received Tocilizumab followed by IVIG had a significantly higher rate of mortality as compared to Tocilizumab alone treated group. Patients who received Tocilizumab followed by IVIG were having more days on mechanical ventilation and spent more days in ICU as compared to Tocilizumab alone treated group. This is in contrast to evidence presented by studies done before that IVIG administration improved outcomes significantly [28-30].

\section{Conclusion}

In our study the group of patients who received Tocilizumab only treatment had better out come in term of survival rate, mortality, need of mechanical ventilation and total number of days on mechanical ventilation, as compare to the other group who received Tocilizumab followed by IVIG therapy. The reason and pathophysiology behind this is not clear However, more studies are needed to make a definitive statement on this regimen's use in the ongoing pandemic.

\section{References}

[1] World Health Organization (WHO) WHO; Geneva, Switzerland: 2020. Coronavirus disease 2019 (COVID-19). Situation report - 51. https://www.who.int/docs/defaultsource/coronaviruse/situation-reports/20200311-sitrep-51-covi d-19. pdf?sfvrsn=1ba62e57_10 [Accessed 1 October 2020] J. Clerk Maxwell, A Treatise on Electricity and Magnetism, 3rd ed., vol. 2. Oxford: Clarendon, 1892, pp. 68-73.

[2] Worldometers. COVID-19 Coronavirus pandemic. Available at https://www.worldometers.info/coronavirus [Accessed 1 October 2020].

[3] Chen J, Lu H, Melino G, et al. COVID-19 infection: the China and Italy perspectives. Cell Death Dis. 2020; 11 (6): 438.
Published 2020 Jun 8. doi: 10.1038/s41419-020-2603-0.

[4] Porcheddu R, Serra C, Kelvin D, Kelvin N, Rubino S. Similarity in Case Fatality Rates (CFR) of COVID-19/SARS-COV-2 in Italy and China. J Infect Dev Ctries. 2020 Feb 29; 14 (2): 125-128. doi: 10.3855/jidc.12600. PMID: 32146445.

[5] Onder G., Rezza G., Brusaferro S. Case-fatality rate and characteristics of patients dying in relation to COVID-19 in Italy. JAMA. 2020 doi: 10.1001/jama.2020.4683.

[6] Zhang Z, Yao W, Wang Y, Long C, Fu X. Wuhan and Hubei COVID-19 mortality analysis reveals the critical role of timely supply of medical resources. J Infect. 2020; 81 (1): 147-178. doi: 10.1016/j.jinf.2020.03.018.

[7] Jean SS, Lee PI, Hsueh PR. Treatment options for COVID-19: The reality and challenges. J Microbiol Immunol Infect. 2020; 53 (3): 436-443. doi: 10.1016/j.jmii.2020.03.034.

[8] Sultana J, Cutroneo PM, Crisafulli S, Puglisi G, Caramori G, Trifirò G. Azithromycin in COVID-19 Patients: Pharmacological Mechanism, Clinical Evidence and Prescribing Guidelines. Drug Saf 2020; 43 (8): 691-698. doi: 10.1007/s40264-020-00976-7.

[9] Ramireddy A, Chugh H, Reinier K, et al. Experience With Hydroxychloroquine and Azithromycin in the Coronavirus Disease 2019 Pandemic: Implications for QT Interval Monitoring. J Am Heart Assoc. 2020; 9 (12): e017144. doi: 10.1161/JAHA.120.017144.

[10] Zhang XL, Li Z. M, Ye JT, et al. Pharmacological and cardiovascular perspectives on the treatment of COVID-19 with chloroquine derivatives [published online ahead of print, 2020 Sep 23]. Acta Pharmacol Sin. 2020; 1-10. doi: 10.1038/s41401-020-00519-x.

[11] Sharma A. Chloroquine paradox may cause more damage than help fight COVID-19. Microbes Infect. 2020; 22 (4-5): 154-156. doi: 10.1016/j.micinf.2020.04.004.

[12] Wong C. K., Lam C. W. K., Wu A. K. L. Plasma inflammatory cytokines and chemokines in severe acute respiratory syndrome. Clin Exp Immunol. 2004; 136 (1): 95-103.

[13] Bhatraju PK, Ghassemieh BJ, Nichols M. Covid-19 in critically ill patients in the Seattle region-case series. N Engl J Med. 2020 doi: 10.1056/NEJMoa2004500. published online March 30 .

[14] Grein J, Ohmagari N, Shin D. Compassionate use of remdesivir for patients with severe Covid-19. N Engl J Med. 2020 doi: 10.1056/NEJMoa2007016. published online April 10.

[15] Holshue ML, DeBolt C, Lindquist S. First case of 2019 novel coronavirus in the United States. N Engl J Med. 2020; 382: 929-936.

[16] Schoot TS, Kerckhoffs APM, Hilbrands LB, van Marum RJ. Immunosuppressive Drugs and COVID-19: A Review. Front Pharmacol. 2020; 11: 1333. Published 2020 Aug 28. doi: 10.3389/fphar.2020.01333

[17] Mastroianni A, Greco S, Apuzzo G, et al. Subcutaneous tocilizumab treatment in patients with severe COVID-19related cytokine release syndrome: an observational cohort study. EClinical Medicine. 2020. doi. org/10.1016/j.eclinm.2020.100410. 
[18] Radbel J, Narayanan N, Bhatt PJ. Use of Tocilizumab for COVID-19-Induced Cytokine Release Syndrome: A Cautionary Case Report. Chest. 2020; 158 (1): e15-e19. doi: 10.1016/j. chest.2020.04.024.

[19] Schwab I, Nimmerjahn F. Intravenous immunoglobulin therapy: how does IgG modulate the immune system? Nat Rev Immunol. 2013 Mar; 13 (3): 176-89. doi: 10.1038/nri3401. Epub 2013 Feb 15. PMID: 23411799.

[20] Kaveri SV, Dietrich G, Hurez V, Kazatchkine MD. Intravenous immunoglobulins (IVIg) in the treatment of autoimmune diseases [published correction appears in Clin Exp Immunol 1992 May; 88 (2): 373]. Clin Exp Immunol. 1991; 86 (2): 192-198. doi: 10.1111/j.1365-2249.1991.tb05794.x.

[21] Zeng F, Huang Y, Guo Y, et al. Association of inflammatory markers with the severity of COVID-19: A meta-analysis. Int J Infect Dis. 2020; 96: 467-474. doi: 10.1016/j.ijid.2020.05.055.

[22] Singh AK, Majumdar S, Singh R, Misra A. Role of corticosteroid in the management of COVID-19: A systemic review and a Clinician\&\#39; s perspective. Diabetes Metab Syndr. 2020; 14 (5): 971-978. doi: 10.1016/j. dsx.2020.06.054.

[23] Modell V, Gee B, Lewis DB, et al. Global study of primary immunodeficiency diseases (PI) - diagnosis, treatment, and economic impact: an updated report from the Jeffrey Modell Foundation. Immunol Res. 2011; 51 (1): 61-70.

[24] Takashi T, Hiroki M, Kiyohide F. Intravenous immunoglobulin and mortality in pneumonia patients with septic shock: an observational nationwide study. Clin Infect Diseases. 2015; 61 (3): 385-392.

[25] Stockman LJ, Bellamy R, Garner P. SARS: Systematic Review of Treatment Effects. PLoS Med. 2006; 3 (9): e343.

[26] Mustafa S, Balkhy H, Gabere MN. Current treatment options and the role of peptides as potential therapeutic components for Middle East Respiratory Syndrome (MERS): A review. J Infect Publ Health. 2017; 11 (1): 9-17.

[27] Ivan F. N, Hung Hyperimmune IV Immunoglobulin treatment: a multicenter double-blind randomized controlled trial for patients with severe 2009 influenza A (H1N1) infection. Chest. 2013; 144 (2): 464-473.

[28] Xie Y, Cao S, Dong H, et al. Effect of regular intravenous immunoglobulin therapy on prognosis of severe pneumonia in patients with COVID-19. J Infect. 2020; 81 (2): 318-356. doi: 10.1016/j.jinf.2020.03.044.

[29] Cao W, et al. High-dose intravenous immunoglobulin as a therapeutic option for deteriorating patients with coronavirus disease 2019. Open Forum Infect Dis. 2020; 7 (3): ofaa102.

[30] Lanza M, Polistina GE, Imitazione $P$, et al. Successful intravenous immunoglobulin treatment in severe COVID-19 pneumonia. IDCases. 2020; 21: e00794. Published 2020 May 16. doi: 10.1016/j.idcr.2020.e00794. 